\title{
Colorings of Periodic Homeomorphisms
}

by

\author{
Yuji AKAIKE, Naotsugu CHINEN and Kazuo TOMOYASU \\ Presented by Czestaw BESSAGA
}

Summary. We calculate the exact value of the color number of a periodic homeomorphism without fixed points on a finite connected graph.

1. Introduction. All spaces are assumed separable metrizable and all maps are continuous. We denote the set of natural numbers by $\mathbb{N}$ and write the unit interval $[0,1]$ as $\mathbb{I}$. If $f: X \rightarrow X$ is a map, then we write inductively $f^{0}=\operatorname{id}_{X}$ and $f^{n}=f \circ f^{n-1}$ for each $n \in \mathbb{N}$.

Let $f: X \rightarrow X$ be a fixed-point free map. A closed subset $A$ of $X$ is called a color of $(X, f)$ if $f(A) \cap A=\emptyset$. A coloring of $(X, f)$ is a finite cover $\mathcal{U}$ of $X$ consisting of colors. The minimal cardinality of a coloring is called the color number of $(X, f)$, denoted by $\operatorname{col}(X, f)$, i.e.,

$$
\operatorname{col}(X, f)=\min \{|\mathcal{U}| \mid \mathcal{U} \text { is a coloring of }(X, f)\} .
$$

Since finite open covers can be shrunk to closed covers, and finite closed covers can be swelled to open covers, the closedness of the coloring is irrelevant. Finite open covers do equally well.

Let $X$ be a set and $f: X \rightarrow X$ a fixed-point free map. It is known that $X$ is the union of disjoint subsets $X_{1}, X_{2}, X_{3}$ such that $f\left(X_{i}\right) \cap X_{i}=\emptyset$ for $i=1,2,3$ (cf. [4], [7]). Subsequently, Błaszczyk and Kim proved the following topological version of the above.

THEOREM 1.1 ([3]). Let $X$ be a 0-dimensional paracompact space and $f: X \rightarrow X$ a fixed-point free homeomorphism. Then $X$ is the union of disjoint clopen sets $X_{1}, X_{2}, X_{3}$ such that $f\left(X_{i}\right) \cap X_{i}=\emptyset$ for $i=1,2,3$.

2000 Mathematics Subject Classification: Primary 54C05; Secondary 54F45.

Key words and phrases: coloring, periodic homeomorphism. 
On the other hand, van Douwen proved that any fixed-point free autohomeomorphism on a finite-dimensional paracompact space has finite color number (cf. [5]). Moreover, an upper bound of the color number was established as follows.

TheOREm 1.2 ([6, Theorem 3]). Let $X$ be a paracompact Hausdorff space with $\operatorname{dim} X \leq n$. If $f: X \rightarrow X$ is a fixed-point free homeomorphism, then $\operatorname{col}(X, f) \leq n+3$.

In [8, Theorem 1.1], J. van Mill gives a simple proof of the theorem above. Furthermore, for a fixed-point free involution, the upper bound of the color number can be improved.

TheOREM 1.3 ([1, Theorem 2]). Let $X$ be a paracompact Hausdorff space with $\operatorname{dim} X \leq n$ and $f: X \rightarrow X$ a fixed-point free homeomorphism. If $f$ is an involution, i.e., $f^{2}(x)=x$ for all $x \in X$, then $\operatorname{col}(X, f) \leq n+2$.

For example, the color number of the rotation through $2 \pi / 3$ on a circle is 4. Moreover, let $S_{Y}^{n}$ be the $n$-dimensional $Y$-sphere and $\gamma^{n+1}: S_{Y}^{n} \rightarrow S_{Y}^{n}$ the period 3 homeomorphism defined in [2, p. 258]. Then $\operatorname{col}\left(S_{Y}^{n}, \gamma^{n+1}\right)=$ $n+3$ ([2, Theorem 4]). Here, $S_{Y}^{1}$ is the bipartite cubic graph $K(3,3)$ on six nodes.

Now, let $X$ be a connected space and $f: X \rightarrow X$ a fixed-point free homeomorphism. Clearly, $\operatorname{col}(X, f) \geq 3$. By Theorem 1.2 , it is natural to ask whether $\operatorname{col}(X, f)=n+3$ or not. In this paper, we concentrate on the following question.

QUESTION 1.4. Let $X$ be a finite connected graph, i.e., a 1-dimensional connected finite simplicial complex, and $f: X \rightarrow X$ a fixed-point free homeomorphism on $X$. Which is true, $\operatorname{col}(X, f)=3$ or $\operatorname{col}(X, f)=4$ ?

We calculate the exact values of the color numbers of certain periodic homeomorphisms. First, we show that if a fixed-point free homeomorphism on an arcwise-connected space has a point of period 3 , then its color number is at least 4 . Next, we calculate the exact value of the color number of a fixedpoint free homeomorphism which has no period 3 point on a finite connected graph: Let $f: X \rightarrow X$ be a fixed-point free homeomorphism with a periodic point on a finite connected graph $X$ and $n_{x}=\min \left\{m \mid f^{m}(x)=x\right\}$. If the greatest common divisor of $\left\{n_{x}\right\}$ is neither 1 nor 3 , then $\operatorname{col}(X, f)=3$.

\section{Fixed-point free homeomorphisms with a period three point.} Let $X$ be a connected space and $f: X \rightarrow X$ a fixed-point free homeomorphism. Clearly, $\operatorname{col}(X, f) \geq 3$. Moreover, if $f^{3}(x)=x$ for each $x \in X$, then $\operatorname{col}(X, f) \geq 4$ (cf. [1, Example 7(1)]). In fact, suppose that there is a coloring $\left\{U_{1}, U_{2}, U_{3}\right\}$ of $(X, f)$. We may assume that $U_{1} \cap U_{2} \neq \emptyset$, and let 
$a \in U_{1} \cap U_{2}$. Then we have $f(a) \in U_{3}$, so $f^{2}(a) \in U_{1} \cup U_{2}$. However, since $f^{3}(a)=a \in U_{1} \cap U_{2}$, we have a contradiction.

The next proposition asserts that if a fixed-point free homeomorphism on an arcwise-connected space with $f^{n}=\mathrm{id}_{X}$ for some $n \in \mathbb{N}$ has a point of period 3 then its color number is at least 4 .

Proposition 2.1. Let $X$ be an arcwise-connected space and $f: X \rightarrow X$ a fixed-point free homeomorphism with $f^{n}=\operatorname{id}_{X}$ for some $n \in \mathbb{N}$. If $f$ has a period 3 point in $X$, then $\operatorname{col}(X, f) \geq 4$.

Proof. On the contrary, suppose that there exists a closed coloring $\mathcal{U}=$ $\left\{U_{1}, U_{2}, U_{3}\right\}$ of $(X, f)$. Let $x$ be a period 3 point for $f$ in $X$, i.e., $f^{3}(x)=x$. Since no two elements of $\left\{x, f(x), f^{2}(x)\right\}$ belong to only one element of $\mathcal{U}$, we may assume that $f^{p-1}(x) \in \operatorname{Int}_{X} U_{p}$ for each $p=1,2,3$ and $f^{p-1}(x) \notin U_{q}$ whenever $p \neq q$.

Let $a \in X \backslash U_{1}$. Since $X$ is arcwise-connected, there exists an embedding $\varphi: \mathbb{I} \rightarrow X$ such that $\varphi(0)=x$ and $\varphi(1)=a$. Let $t_{0}=\inf \left\{s \in \mathbb{I} \mid \varphi(s) \notin U_{1}\right\}$. Then $\varphi\left(t_{0}\right) \in U_{2} \cup U_{3}$.

Assume that $\varphi\left(t_{0}\right) \in U_{2}$. Since $\mathcal{U}$ is a coloring, $f\left(\varphi\left(t_{0}\right)\right) \notin U_{2}$. Let $t_{1}=$ $\inf \left\{s \in \mathbb{I} \mid f(\varphi(s)) \notin U_{2}\right\}$. Note that $t_{1}<t_{0}$, and thus $f\left(\varphi\left(\left[0, t_{1}\right]\right)\right) \subsetneq$ $f\left(\varphi\left(\left[0, t_{0}\right]\right)\right)$. Moreover, we have $f\left(\varphi\left(\left[0, t_{0}\right]\right)\right) \cap U_{1}=\emptyset$ and $f\left(\varphi\left(t_{1}\right)\right) \in U_{3}$. Since $\mathcal{U}$ is a coloring, $f^{2}\left(\varphi\left(t_{1}\right)\right) \notin U_{3}$. Let $t_{2}=\inf \left\{s \in \mathbb{I} \mid f^{2}(\varphi(s)) \notin U_{3}\right\}$. As $t_{2}<t_{1}$, we have $f^{2}\left(\varphi\left(\left[0, t_{2}\right]\right)\right) \subsetneq f^{2}\left(\varphi\left(\left[0, t_{1}\right]\right)\right), f^{2}\left(\varphi\left(\left[0, t_{1}\right]\right)\right) \cap U_{2}=\emptyset$, and $f^{2}\left(\varphi\left(t_{2}\right)\right) \in U_{1}$. Continuing in this fashion, we obtain

$$
\begin{aligned}
& f^{n-1}\left(\varphi\left(t_{n-2}\right)\right) \notin U_{3}, \quad f^{n-1}\left(\varphi\left(t_{n-1}\right)\right) \in U_{1}, \\
& f^{n-1}\left(\varphi\left(\left[0, t_{n-1}\right]\right)\right) \subsetneq f^{n-1}\left(\varphi\left(\left[0, t_{n-2}\right]\right)\right), \quad \text { and } t_{n-1}<t_{n-2} .
\end{aligned}
$$

Let $t_{n}=\inf \left\{s \in \mathbb{I} \mid f^{n}(\varphi(s)) \notin U_{1}\right\}$. Since $t_{n}<t_{n-1}$, it follows that $f^{n}\left(\varphi\left(\left[0, t_{n}\right]\right)\right) \subsetneq f^{n}\left(\varphi\left(\left[0, t_{n-1}\right]\right)\right)$. On the other hand, by the definition of $t_{n}$ and $f^{n}=\mathrm{id}_{X}$, we obtain $t_{n}=t_{0}$, a contradiction.

If $\varphi\left(t_{0}\right) \in U_{3}$, mimicking the argument above, we also have a contradiction.

By Theorem 1.2 and Proposition 2.1, we have the following.

Corollary 2.2. Let $X$ be a 1-dimensional arcwise-connected space and $f: X \rightarrow X$ a fixed-point free homeomorphism with $f^{n}=\operatorname{id}_{X}$ for some $n \in \mathbb{N}$. If $f$ has a period 3 point in $X$, then $\operatorname{col}(X, f)=4$.

EXAMPLE 2.3. Let $Z_{n}=\left\{x_{0}, x_{1}, \ldots, x_{n-1}\right\}$ be an $n$-point discrete space, and $Z_{m} * Z_{n}$ a join of $Z_{m}$ and $Z_{n}$. Define $f_{n}: Z_{n} \rightarrow Z_{n}$ by $f_{n}\left(x_{i}\right)=x_{i+1} \bmod$ ulo $n$ for $i=0, \ldots, n-1$, and let $f_{m} * f_{n}: Z_{m} * Z_{n} \rightarrow Z_{m} * Z_{n}$ be the natural map constructed from $f_{m}$ and $f_{n}$. By Corollary $2.2, \operatorname{col}\left(Z_{3} * Z_{n}, f_{3} * f_{n}\right)$ $=4$ for all $n \in \mathbb{N}$ with $n \geq 2$. 
3. Fixed-point free homeomorphisms without period three point. In this section, we calculate the exact value of the color number for a fixed-point free homeomorphism without period 3 points on a finite connected graph.

Let $\mathcal{U}=\left\{U_{1}, \ldots, U_{p}\right\}$ be a coloring of $(X, f)$. If we wish to emphasize the number of colors of $\mathcal{U}$, we say that $\mathcal{U}$ is a p-coloring of $(X, f)$.

Definition 3.1. Let $f: X \rightarrow X$ be a map, $A$ a closed subset of $X$ with $f(A) \subset A$, and $\mathcal{U}=\left\{U_{1}, \ldots, U_{p}\right\}$ a $p$-coloring of $\left(A,\left.f\right|_{A}\right)$. We say that a coloring $\widetilde{\mathcal{U}}=\left\{\widetilde{U}_{1}, \ldots, \widetilde{U}_{q}\right\}(p \leq q)$ of $(X, f)$ is an extension of $\mathcal{U}$ to $(X, f)$, or $\mathcal{U}$ extends to the q-coloring $\widetilde{\mathcal{U}}$ of $(X, f)$ if $\widetilde{U}_{i} \cap A=U_{i}$ for each $i \leq p$.

We denote by $\langle n\rangle=\{0,1, \ldots, n-1\}$ the cyclic additive group with 0 the unit element. Let $X_{n}=\mathbb{I} \times\langle n\rangle, B_{n}=\{0,1\} \times\langle n\rangle$, and $f_{n}: X_{n} \rightarrow X_{n}$ be a homeomorphism which is represented by $f_{n}(t, i)=\left(f_{n, i}(t), i+1\right)$ with addition modulo $n$, where $f_{n, i}: \mathbb{I} \rightarrow \mathbb{I}$ is an order preserving homeomorphism for each $i=0,1, \ldots, n-1$. Note that $f_{n}^{p}(\delta, i)=(\delta, i+p)=f_{n}^{i}(\delta, p)$ for each $\delta=0,1$.

Let $f: X \rightarrow X$ be a map. Then the mapping torus of $(X, f)$, written $M(X, f)$, is obtained from $X \times \mathbb{I}$ by identifying $(x, 1)$ with $(f(x), 0)$. Let $s_{n}:\langle n\rangle \rightarrow\langle n\rangle$ be the shift map, i.e., $s_{n}(i)=i+1$. Then $s_{n}$ has a natural extension $\widetilde{s}_{n}$ on $M\left(\langle n\rangle, s_{n}\right)$, namely, $\widetilde{s}_{n}(i, t)=\left(s_{n}(i), t\right)$ and the rotation $R_{n}: \mathbb{S}^{1} \rightarrow \mathbb{S}^{1}$ through $2 \pi / n$ can be viewed as $\widetilde{s}_{n}$ on $M\left(\langle n\rangle, s_{n}\right)$.

We will consider extension of colorings of $\left(B_{n},\left.f_{n}\right|_{B_{n}}\right)$ to colorings of $\left(X_{n}, f_{n}\right)$ for $n \geq 2$.

Notation 3.2. Let $j=0,1, k=0, \ldots, n-1$, and $\mathcal{U}=\left\{U_{p} \mid p=1, \ldots, q\right\}$ a coloring of $\left(B_{n},\left.f_{n}\right|_{B_{n}}\right)$. We represent $\mathcal{U}$ by the $2 \times n$ matrix whose $(j+$ $1, k+1)$-component is $p$ if $(j, k) \in U_{p}$.

For example, let $n=3, U_{1}=\{0,1\} \times\{1\}, U_{2}=\{0,1\} \times\{2\}$ and $U_{3}=$ $\{0,1\} \times\{0\}$. Then $\mathcal{U}=\left\{U_{1}, U_{2}, U_{3}\right\}$ is represented by $\left(\begin{array}{lll}3 & 1 & 2 \\ 3 & 1 & 2\end{array}\right)$. Let $n=4$, $V_{1}=\{(0,0),(0,2),(1,1),(1,3)\}$ and $V_{2}=\{(0,1),(0,3),(1,0),(1,2)\}$. Then $\mathcal{V}=\left\{V_{1}, V_{2}\right\}$ is represented by $\left(\begin{array}{llll}1 & 2 & 1 & 2 \\ 2 & 1 & 2 & 1\end{array}\right)$.

Lemma 3.3. Let $n=2 m$ with $m \geq 1$ and $\mathcal{U}^{(n)}=\left\{U_{1}, U_{2}\right\}$ a 2-coloring of $\left(B_{n},\left.f_{n}\right|_{B_{n}}\right)$.

(1) The 2-coloring $\mathcal{U}^{(n)}=\left(\begin{array}{ccccc}1 & 2 & \cdots & 1 & 2 \\ 1 & 2 & \cdots & 1 & 2\end{array}\right)$ of $\left(B_{n},\left.f_{n}\right|_{B_{n}}\right)$ extends to a 2-coloring $\widetilde{\mathcal{U}}^{(n)}$ of $\left(X_{n}, f_{n}\right)$.

(2) The 2-coloring $\mathcal{U}^{(n)}=\left(\begin{array}{lllll}1 & 2 & \cdots & 1 & 2 \\ 2 & 1 & \ldots & 2 & 1\end{array}\right)$ of $\left(B_{n},\left.f_{n}\right|_{B_{n}}\right)$ extends to a 3-coloring $\widetilde{\mathcal{U}}^{(n)}$ of $\left(X_{n}, f_{n}\right)$. 
Proof. (1) Let

$$
\widetilde{U}_{1}=\bigcup_{i=0}^{m-1} \mathbb{I} \times\{2 i\} \quad \text { and } \quad \widetilde{U}_{2}=\bigcup_{i=0}^{m-1} \mathbb{I} \times\{2 i+1\} .
$$

Since $\widetilde{U}_{p} \cap B_{n}=U_{p}$ for $p=1,2$, the covering $\widetilde{\mathcal{U}}=\left\{\widetilde{U}_{1}, \widetilde{U}_{2}\right\}$ is as desired.

(2) Let $x_{0}=(1 / 2,0), y_{0}=1 / 2, x_{i}=f_{n}^{i}\left(x_{0}\right)=\left(y_{i}, i\right)$ for each $i=$ $1, \ldots, 2 m-1$, and $x_{2 m-1}^{\prime}=f_{n}^{-1}\left(x_{0}\right)=\left(y_{2 m-1}^{\prime}, 2 m-1\right)$. Let

$$
\begin{aligned}
\widetilde{U}_{1}= & \left(\bigcup_{i=0}^{m-1}\left[0, y_{2 i}\right] \times\{2 i\}\right) \cup\left(\bigcup_{i=0}^{m-2}\left[\frac{1}{2}\left(1+y_{2 i+1}\right), 1\right] \times\{2 i+1\}\right) \\
& \cup\left[\frac{1}{2}\left(1+\max \left\{y_{2 m-1}, y_{2 m-1}^{\prime}\right\}\right), 1\right] \times\{2 m-1\}, \\
\widetilde{U}_{2}= & \left(\bigcup_{i=0}^{m-1}\left[y_{2 i}, 1\right] \times\{2 i\}\right) \cup\left(\bigcup_{i=0}^{m-2}\left[0, \frac{y_{2 i+1}}{2}\right] \times\{2 i+1\}\right) \\
& \cup\left[0, \frac{1}{2} \min \left\{y_{2 m-1}, y_{2 m-1}^{\prime}\right\}\right] \times\{2 m-1\}, \\
\widetilde{U}_{3}= & \left(\bigcup_{i=0}^{m-2}\left[\frac{y_{2 i+1}}{2}, \frac{1}{2}\left(1+y_{2 i+1}\right)\right] \times\{2 i+1\}\right) \\
& \cup\left[\frac{\min \left\{y_{2 m-1}, y_{2 m-1}^{\prime}\right\}}{2}, \frac{1}{2}\left(1+\max \left\{y_{2 m-1}, y_{2 m-1}^{\prime}\right\}\right)\right] \times\{2 m-1\} .
\end{aligned}
$$

The covering $\widetilde{\mathcal{U}}=\left\{\widetilde{U}_{1}, \widetilde{U}_{2}, \widetilde{U}_{3}\right\}$ is as desired.

Fix $n=2 m+1$ and $l=0,1, \ldots, n-1$. Define the 3-coloring $\mathcal{U}^{(l, n)}$ of $\left(B_{n},\left.f_{n}\right|_{B_{n}}\right)$ to be $\mathcal{U}^{(l, n)}=\left\{U_{1}^{(l, n)}, U_{2}^{(l, n)}, U_{3}^{(l, n)}\right\}$, where

$$
\begin{aligned}
& U_{1}^{(l, n)}=\{(0,2 i+1),(1, l+2 i+1) \mid i=0,1, \ldots, m-1\}, \\
& U_{2}^{(l, n)}=\{(0,2 i+2),(1, l+2 i+2) \mid i=0,1, \ldots, m-1\}, \\
& U_{3}^{(l, n)}=\{(0,0),(1, l)\} .
\end{aligned}
$$

For any $k=1,2, \mathcal{U}^{(k, 3)}$ does not extend to a 3-coloring of $\left(X_{3}, f_{3}\right)$. In fact, the existence of an extended 3-coloring $\widetilde{\mathcal{U}}^{(k, 3)}$ of $\left(M\left(\langle 3\rangle, s_{3}\right), \widetilde{s}_{3}\right)$ which is the rotation through $2 \pi / 3$ on the circle contradicts Proposition 2.1. Thus, in the rest of this paper, we only consider the case where $m \geq 2$.

We define the map $\varphi:\{0,1\} \times\langle n\rangle \rightarrow\{0,1\} \times\langle n\rangle$ by $\varphi(t, i)=(1-t, i)$.

Lemma 3.4. For any $p=1,2,3, f_{n}^{n-l} \circ \varphi\left(U_{p}^{(l, n)}\right)=U_{p}^{(n-l, n)}$. 
Proof. We can calculate as follows:

$$
\begin{gathered}
f_{n}^{n-l} \circ \varphi\left(U_{1}^{(l, n)}\right)=f_{n}^{n-l}(\{(1,2 i+1),(0, l+2 i+1) \mid i=0,1, \ldots, m-1\}) \\
=\{(1,2 i+1+n-l),(0, l+2 i+1+n-l) \mid i=0,1, \ldots, m-1\} \\
=\{(0,2 i+1),(1, n-l+2 i+1) \mid i=0,1, \ldots, m-1\}=U_{1}^{(n-l, n)}, \\
f_{n}^{n-l} \circ \varphi\left(U_{2}^{(l, n)}\right)=f_{n}^{n-l}(\{(1,2 i+2),(0, l+2 i+2) \mid i=0,1, \ldots, m-1\}) \\
=\{(1,2 i+2+n-l),(0, l+2 i+2+n-l) \mid i=0,1, \ldots, m-1\} \\
=\{(0,2 i+2),(1, n-l+2 i+2) \mid i=0,1, \ldots, m-1\}=U_{2}^{(n-l, n)}, \\
f_{n}^{n-l} \circ \varphi\left(U_{3}^{(l, n)}\right)=f_{n}^{n-l}(\{(1,0),(0, l)\})=\{(1, n-l),(0, l+n-l)\} \\
=\{(0,0),(1, n-l)\}=U_{3}^{(n-l, n)} .
\end{gathered}
$$

REMARK 3.5. By the lemma above, if $\mathcal{U}^{(l, n)}$ extends to a coloring of $\left(X_{n}, f_{n}\right)$, then so does $\mathcal{U}^{(n-l, n)}$ for $1 \leq l \leq n-1$. Note that $\mathcal{U}^{(0, n)}$ extends to a 3 -coloring of $\left(X_{n}, f_{n}\right)$. In fact, let $\widetilde{U}_{1}^{(0, n)}=\bigcup_{i=0}^{m-1} \mathbb{I} \times\{2 i+1\}$, $\widetilde{U}_{2}^{(0, n)}=\bigcup_{i=0}^{m-1} \mathbb{I} \times\{2 i+2\}$, and $\widetilde{U}_{3}^{(0, n)}=\mathbb{I} \times\{0\}$. Then the coloring $\widetilde{\mathcal{U}}^{(0, n)}=\left\{\widetilde{U}_{1}^{(0, n)}, \widetilde{U}_{2}^{(0, n)}, \widetilde{U}_{3}^{(0, n)}\right\}$ of $\left(X_{n}, f_{n}\right)$ extends $\mathcal{U}^{(0, n)}$. Hence, we need only check whether $\mathcal{U}^{(l, n)}$ extends to a 3 -coloring of $\left(X_{n}, f_{n}\right)$ for $l=1, \ldots, m$ and $n=2 m+1$ with $m \geq 2$.

Notation 3.6. Let $\widetilde{\mathcal{U}}^{(m)}=\left\{U_{1}^{m}, U_{2}^{m}, U_{3}^{m}\right\}$ and $\widetilde{\mathcal{U}}^{(n)}=\left\{U_{1}^{n}, U_{2}^{n}, U_{3}^{n}\right\}$ be covers of $X_{m}$ and $X_{n}$, respectively. Define a cover $\widetilde{\mathcal{U}}^{(m)}+\widetilde{\mathcal{U}}^{(n)}=$ $\left\{U_{1}^{m+n}, U_{2}^{m+n}, U_{3}^{m+n}\right\}$ of $X_{m+n}$ by $U_{i}^{m+n}=U_{i}^{m} \cup f_{m+n}^{m}\left(U_{i}^{n}\right)$ for $i=1,2,3$.

Lemma 3.7. Let $n=2 m+1$ with $m \geq 2$. Then the 3 -colorings $\mathcal{U}^{(1, n)}$ and $\mathcal{U}^{(2, n)}$ extend to 3 -colorings of $\left(X_{n}, f_{n}\right)$.

Proof. We note that

$$
\mathcal{U}^{(1, n)}=\left(\begin{array}{llllll}
3 & 1 & 2 & \cdots & 1 & 2 \\
2 & 3 & 1 & \cdots & 2 & 1
\end{array}\right)
$$

can be considered as

$$
\left(\begin{array}{lllllllll}
2 & 1 & \cdots & 1 & 2 & 3 & 1 & 2 & 1 \\
1 & 2 & \cdots & 2 & 1 & 2 & 3 & 1 & 2
\end{array}\right) .
$$

Let $x_{0}=(1 / 2,0)$ and $x_{i}=f_{n}^{i}\left(x_{0}\right)=\left(y_{i}, i\right)$ for each $i=0, \ldots, 2 m-3$. Set $y_{2 m-3}^{\prime}=\left(1+y_{2 m-3}\right) / 2, y_{2 m-2} \in \mathbb{I}$ with $f_{n}\left(y_{2 m-3}^{\prime}, 2 m-3\right)=\left(y_{2 m-2}, 2 m-2\right)$, $y_{2 m-2}^{\prime}=\left(1+y_{2 m-2}\right) / 2, y_{2 m-1} \in \mathbb{I}$ with $f_{n}\left(y_{2 m-2}^{\prime}, 2 m-2\right)=\left(y_{2 m-1}, 2 m-1\right)$, $y_{2 m-1}^{\prime}=\left(1+y_{2 m-1}\right) / 2, y_{2 m} \in \mathbb{I}$ with $f_{n}\left(y_{2 m-1}^{\prime}, 2 m-1\right)=\left(y_{2 m}, 2 m\right)$, 
and $y_{2 m}^{\prime \prime} \in \mathbb{I}$ with $f_{n}^{-1}\left(x_{0}\right)=\left(y_{2 m}^{\prime \prime}, 2 m\right)$. Let

$$
\begin{aligned}
\widetilde{U}_{1}= & \left(\bigcup_{i=0}^{m-2}\left[y_{2 i}, 1\right] \times\{2 i\} \cup \bigcup_{i=0}^{m-3}\left[0, \frac{y_{2 i+1}}{2}\right] \times\{2 i+1\}\right) \\
& \cup\left[0, y_{2 m-2}^{\prime}\right] \times\{2 m-2\} \cup\left[y_{2 m-1}^{\prime}, 1\right] \times\{2 m-1\} \\
& \cup\left[0, \frac{1}{2} \min \left\{y_{2 m}, y_{2 m}^{\prime \prime}\right\}\right] \times\{2 m\}, \\
\widetilde{U}_{2}= & \left(\bigcup_{i=0}^{m-2}\left[0, y_{2 i}\right] \times\{2 i\} \cup \bigcup_{i=0}^{m-3}\left[\frac{1}{2}\left(1+y_{2 i+1}\right), 1\right] \times\{2 i+1\}\right) \\
& \cup\left[y_{2 m-3}^{\prime}, 1\right] \times\{2 m-3\} \cup\left[0, y_{2 m-1}^{\prime}\right] \times\{2 m-1\} \\
& \cup\left[\frac{1}{2}\left(1+\max \left\{y_{2 m}, y_{2 m}^{\prime \prime}\right\}\right), 1\right] \times\{2 m\}, \\
\widetilde{U}_{3}= & \left(\bigcup_{i=0}^{m-3}\left[\frac{y_{2 i+1}}{2}, \frac{1}{2}\left(1+y_{2 i+1}\right)\right] \times\{2 i+1\}\right) \\
& \cup\left[0, y_{2 m-3}^{\prime}\right] \times\{2 m-3\} \cup\left[y_{2 m-2}^{\prime}, 1\right] \times\{2 m-2\} \\
& \cup\left[\frac{1}{2} \min \left\{y_{2 m}, y_{2 m}^{\prime \prime}\right\}, \frac{1}{2}\left(1+\max \left\{y_{2 m}, y_{2 m}^{\prime \prime}\right\}\right)\right] \times\{2 m\} .
\end{aligned}
$$

Then $\widetilde{\mathcal{U}}=\left\{\widetilde{U}_{1}, \widetilde{U}_{2}, \widetilde{U}_{3}\right\}$ is a 3 -coloring of $\left(X_{2 m+1}, f_{2 m+1}\right)$ extending

$$
\mathcal{U}^{(1, n)}=\left(\begin{array}{lllllllll}
2 & 1 & \cdots & 1 & 2 & 3 & 1 & 2 & 1 \\
1 & 2 & \cdots & 2 & 1 & 2 & 3 & 1 & 2
\end{array}\right) .
$$

Next, we consider the 3 -coloring

$$
\mathcal{U}^{(2, n)}=\left(\begin{array}{lllllll}
3 & 1 & 2 & 1 & 2 & \cdots & 2 \\
1 & 2 & 3 & 1 & 2 & \cdots & 2
\end{array}\right) .
$$

Let $x_{0}=(1 / 2,0)=\left(y_{0}, 0\right), x_{1}=f_{n}\left(x_{0}\right)=\left(y_{1}, 1\right), x_{2}=f_{n}\left(y_{1} / 2,1\right)=\left(y_{2}, 2\right)$,

$$
\begin{aligned}
& \widetilde{V}_{1}^{3}=\left[y_{0}, 1\right] \times\{0\} \cup\left[0, y_{1} / 2\right] \times\{1\}, \\
& \widetilde{V}_{2}^{3}=\left[y_{1} / 2,1\right] \times\{1\} \cup\left[0, y_{2} / 2\right] \times\{2\}, \\
& \widetilde{V}_{3}^{3}=\left[0, y_{0}\right] \times\{0\} \cup\left[y_{2} / 2,1\right] \times\{2\} .
\end{aligned}
$$

Then $\widetilde{\mathcal{V}}^{(3)}=\left\{\widetilde{V}_{1}^{3}, \widetilde{V}_{2}^{3}, \widetilde{V}_{3}^{3}\right\}$ is a cover of $X_{3}$, and $\widetilde{\mathcal{V}}^{(3)}+\widetilde{\mathcal{U}}^{(2 m-2)}$ is a 3-coloring of $\left(X_{2 m+1}, f_{2 m+1}\right)$ extending $\mathcal{U}^{(2, n)}$, where $\widetilde{\mathcal{U}}^{(2 m-2)}$ is defined as in Lemma 3.3(1).

Lemma 3.8. Let $n=2 m+1$ with $m \geq 2$. Then $\mathcal{U}^{(l, n)}$ extends to 3 coloring $\widetilde{\mathcal{U}}^{(l, n)}$ of $\left(X_{n}, f_{n}\right)$ for each $l=0,1, \ldots, n-1$. 
Proof. By Remark 3.5, we argue by induction on $m$ and $l$ with $l=$ $1, \ldots, m$.

By Lemma 3.7, the coloring $\mathcal{U}^{(l, 5)}$ extends to a 3 -coloring of $\left(X_{5}, f_{5}\right)$ for $m=2$ and $l=1,2$.

Suppose that the coloring

$$
\mathcal{U}^{(l, n)}=\left(\begin{array}{ccccccccc}
3 & 1 & 2 & \cdots & i & j & \cdots & 1 & 2 \\
& & \cdots & 2 & 3 & 1 & \cdots & &
\end{array}\right)
$$

of $\left(B_{n}, f_{n} \mid B_{n}\right)$, represented by a $2 \times n$ matrix whose $(2, l+1)$-component is 3 , extends to a 3-coloring $\widetilde{\mathcal{U}}^{(l, n)}=\left\{\widetilde{U}_{1}^{(l, n)}, \widetilde{U}_{2}^{(l, n)}, \widetilde{U}_{3}^{(l, n)}\right\}$ for each $n \leq 2 m+1$ and each $l=0, \ldots, n-1$. We may assume that $\widetilde{U}_{2}^{(l, n)} \cap(\mathbb{I} \times\{l\})=\emptyset$ and $\widetilde{U}_{1}^{(l, n)} \cap(\mathbb{I} \times\{0\})=\emptyset$ if $(i, j)=(1,2), \widetilde{U}_{1}^{(l, n)} \cap(\mathbb{I} \times\{l\})=\emptyset$ and $\mathbb{I} \times\{n\} \subset \widetilde{U}_{2}^{(l, n)}$ if $(i, j)=(2,1)$, and $\mathbb{I} \times\{k\} \subset \widetilde{U}_{i}^{(l, n)}$ if the $(1, k+1)$-component and $(2, k+1)$ component are equal to $i$.

In the case of $(i, j)=(1,2)$, by Lemma 3.7 , the 3 -coloring $\mathcal{U}^{(1,2 m+3)}$, represented by a $2 \times(2 m+3)$ matrix whose $(2,2)$-component is 3 , extends to a 3 -coloring of $\left(X_{2 m+3}, f_{2 m+3}\right)$. Then we may assume that $l \geq 3$. We will consider the 3 -coloring

$$
\mathcal{U}^{(l, 2 m+3)}=\left(\begin{array}{llllllllllll}
3 & 1 & 2 & \cdots & 2 & 1 & 2 & 1 & 2 & \cdots & 1 & 2 \\
2 & 1 & 2 & \cdots & 2 & 3 & 1 & 2 & 1 & \cdots & 2 & 1
\end{array}\right),
$$

represented by a $2 \times(2 m+3)$ matrix whose $(2, l+1)$-component is 3 , as

$$
\left(\begin{array}{cccccccccccc}
1 & 2 & \cdots & 2 & 1 & 2 & 1 & 2 & \cdots & 1 & 2 & 3 \\
1 & 2 & \cdots & 2 & 3 & 1 & 2 & 1 & \cdots & 2 & 1 & 2
\end{array}\right),
$$

represented by a $2 \times(2 m+3)$ matrix whose $(2, l)$-component is 3 . By induction, the 3-coloring

$$
\mathcal{U}^{(l-2,2 m+1)}=\left(\begin{array}{cccccccccccc}
1 & 2 & \cdots & 2 & 1 & 2 & 1 & 2 & \cdots & 1 & 2 & 3 \\
1 & 2 & \cdots & 2 & 3 & 1 & 2 & 1 & \cdots & 2 & 1 & 2
\end{array}\right),
$$

represented by a $2 \times(2 m+1)$ matrix whose $(2, l-2)$-component is 3 , extends to a 3-coloring $\widetilde{\mathcal{U}}^{(l-2,2 m+1)}$ of $\left(X_{2 m+1}, f_{2 m+1}\right)$. Here, we may assume that $\mathbb{I} \times\{0\} \subset \widetilde{U}_{1}^{(l-2,2 m+1)}$ and $\widetilde{U}_{1}^{(l-2,2 m+1)} \cap(\mathbb{I} \times\{2 m\})=\emptyset$. Let $\widetilde{\mathcal{U}}^{(2)}$ be as in Lemma 3.3(1). Then $\widetilde{\mathcal{U}}^{(2)}+\widetilde{\mathcal{U}}^{(l-2,2 m+1)}$ is a 3-coloring of $\left(X_{2 m+3}, f_{2 m+3}\right)$ extending $\mathcal{U}^{(l, 2 m+3)}$.

In the case of $(i, j)=(2,1)$, by the arguments above, we may assume that $l \geq 3$. By induction, the coloring

$$
\mathcal{U}^{(l, 2 m+1)}=\left(\begin{array}{lllllllllll}
3 & 1 & 2 & \cdots & 1 & 2 & 1 & 2 & \cdots & 1 & 2 \\
1 & 2 & 1 & \cdots & 2 & 3 & 1 & 2 & \cdots & 1 & 2
\end{array}\right),
$$


represented by a $2 \times(2 m+1)$ matrix whose $(2, l+1)$-component is 3 , extends to a 3 -coloring $\widetilde{\mathcal{U}}^{(l, 2 m+1)}$ of $\left(X_{2 m+1}, f_{2 m+1}\right)$ such that $\widetilde{U}_{2}^{(l, 2 m+1)} \cap(\mathbb{I} \times\{0\})=\emptyset$ and $\mathbb{I} \times\{2 m\} \subset \widetilde{U}_{2}^{(l, 2 m+1)}$. Let $\widetilde{\mathcal{U}}^{(2)}$ be as in Lemma 3.3(1). Then $\widetilde{\mathcal{U}}^{(l, 2 m+1)}+$ $\widetilde{\mathcal{U}}^{(2)}$ is a 3 -coloring of $\left(X_{2 m+3}, f_{2 m+3}\right)$ extending $\mathcal{U}^{(l, 2 m+3)}$.

Combining the lemmas above, we have the following.

LEMma 3.9. Let $\mathcal{U}^{(n)}$ and $\mathcal{U}^{(l, n)}$ be colorings as in Lemma 3.3 and 3.8 of $\left(B_{n},\left.f_{n}\right|_{B_{n}}\right)$ with $n \in \mathbb{N} \backslash\{1,3\}$, respectively. Then $\mathcal{U}^{(n)}$ and $\mathcal{U}^{(l, n)}$ extend to 3 -colorings of $\left(X_{n}, f_{n}\right)$ for $l=0,1, \ldots, n-1$.

For any homeomorphism $f: X \rightarrow X$ and any periodic point $x \in X$, we write $n_{x}=\min \left\{m \mid f^{m}(x)=x\right\}$. Set $\mathrm{P}(f)=\{x \mid x$ is a periodic point of $f\}$ and $\mathrm{O}(x)=\left\{x, f(x), \ldots, f^{n_{x}-1}(x)\right\}$.

Proposition 3.10. Let $\mathcal{T}$ be a triangulation of a finite connected graph $X$ and $f: X \rightarrow X$ a fixed-point free homeomorphism with $\mathrm{P}(f) \neq \emptyset$. If there exists an $n \in \mathbb{N} \backslash\{1,3\}$ such that $n_{x}$ is a multiple of $n$ for each $x \in \mathrm{P}(f)$, then $\operatorname{col}(X, f)=3$.

Proof. Since $X$ is connected, $\operatorname{col}(X, f) \geq 3$. We may assume that $\left|\mathcal{T}^{(0)}\right|$ $\subset \mathrm{P}(f)$. We can choose $x_{i} \in\left|\mathcal{T}^{(0)}\right|$ and decompose $\left|\mathcal{T}^{(0)}\right|=\bigcup_{i=1}^{N_{0}} \mathrm{O}\left(x_{i}\right)$. For each $i$, let $m_{i}$ be the number such that $n_{x_{i}}=m_{i} n$.

If $n=2 n^{\prime}$, for each $i=1, \ldots, N_{0}$ let

$$
\begin{aligned}
& \left.U_{i, 1}=\left\{f^{2 p}\left(x_{i}\right) \mid 0 \leq p \leq m_{i} n^{\prime}-1\right)\right\}, \\
& U_{i, 2}=\left\{f^{2 p+1}\left(x_{i}\right) \mid 0 \leq p \leq m_{i} n^{\prime}-1\right\} .
\end{aligned}
$$

For simplicity of notation, as in Notation 3.2, we can write

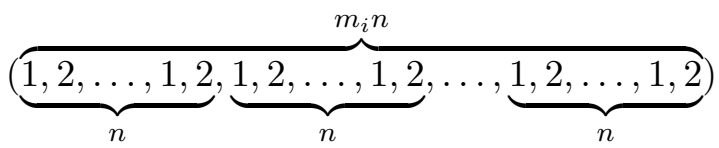

instead of the coloring $\left\{U_{i, 1}, U_{i, 2}\right\}$ of $\mathrm{O}\left(x_{i}\right)$. Let $U_{j}=\bigcup_{i=1}^{N_{0}} U_{i, j}$ for $j=1,2$ and $\mathcal{U}=\left\{U_{1}, U_{2}\right\}$. If $n=2 n^{\prime}+1$, let us denote a coloring of $\mathrm{O}\left(x_{i}\right)$ by

$$
\left\{U_{i, 1}, U_{i, 2}, U_{i, 3}\right\}=(\overbrace{\underbrace{1,2, \ldots, 1,2,3}_{n}}^{1,2, \ldots, 1,2,3}, \ldots, \underbrace{m_{i} n}_{n}, \underbrace{1,2, \ldots, 1,2,3}_{n}),
$$

where $U_{i, j}=\left\{f^{p}\left(x_{i}\right) \mid(1, p+1)\right.$-component is equal to $\left.j\right\}$ for $j=1,2,3$. Let $U_{j}=\bigcup_{i=1}^{N_{0}} U_{i, j}$ for $j=1,2,3$ and $\mathcal{U}=\left\{U_{1}, U_{2}, U_{3}\right\}$. Note that $\mathcal{U}$ is a coloring of $\left(\left|\mathcal{T}^{(0)}\right|,\left.f\right|_{\left|\mathcal{T}^{(0)}\right|}\right)$.

Now, there exist 1 -simplexes $\sigma_{1}, \ldots, \sigma_{N_{1}}$ of $\mathcal{T}$ and $l_{1}, \ldots, l_{N_{1}} \in \mathbb{N}$ such that $\left|\sigma_{k}\right|=f^{p}\left(\left|\sigma_{k}\right|\right)$ for $1 \leq p \leq l_{k} n$ if and only if $p=l_{k} n$. Let $Y_{k}=$ $\bigcup_{p=1}^{l_{k} n} f^{p}\left(\left|\sigma_{k}\right|\right)$ for each $k=1, \ldots, N_{1}$, and thus $X=\bigcup_{k=1}^{N_{1}} Y_{k}$, with $Y_{i}=Y_{j}$ if and only if $i=j$. 
Suppose that there exists an $x \in Y_{k}^{(0)}=\bigcup_{p=1}^{l_{k} n} f^{p}\left(\left|\sigma_{k}^{(0)}\right|\right)$ such that $\mathrm{O}(x)=Y_{k}^{(0)}$. Then, by the fixed point theorem, there exists a $y \in Y_{k} \backslash Y_{k}^{(0)}$ such that $f^{l_{k} n}(y)=y$. In this case, adding $\mathrm{O}(y)$ to $\mathcal{T}^{(0)}$, we may assume that $f^{l_{k} n}(x)=x$ for each $x \in Y_{k}^{(0)}$.

Consider a disjoint sum $X_{l_{k} n}=\bigoplus_{p=1}^{l_{k} n} f^{p}\left(\left|\sigma_{k}\right|\right)$ for each $k=1, \ldots, N_{1}$ and a coloring $\left(X_{l_{k} n}, f_{l_{k} n}\right)$. Let $B_{l_{k} n}=\bigoplus_{p=1}^{l_{k} n} f^{p}\left(\left|\sigma_{k}^{(0)}\right|\right)$. Then the coloring $\mathcal{V}_{k}$ of $\left(B_{l_{k} n},\left.f_{l_{k} n}\right|_{B_{l_{k} n}}\right)$ is naturally induced by $\mathcal{U} \cap Y_{k}^{(0)}$, i.e., there exist an $l$ with $0 \leq l \leq n-1$ and a coloring $\mathcal{W}_{k} \in\left\{\mathcal{U}^{(n)}, \mathcal{U}^{(l, n)}\right\}$ such that

$$
\mathcal{V}_{k}=\underbrace{\mathcal{W}_{k}+\cdots+\mathcal{W}_{k}}_{l_{k}}
$$

where $\mathcal{U}^{(n)}, \mathcal{U}^{(l, n)}$ are as in Lemma 3.9. Thus, $\mathcal{V}_{k}$ extends to a 3 -coloring $\widetilde{\mathcal{V}}_{k}$ of $\left(X_{l_{k} n}, f_{l_{k} n}\right)$. This shows that $\mathcal{U} \cap Y_{k}^{(0)}$ extends to a 3 -coloring $\left\{\widetilde{U}_{k, 1}, \widetilde{U}_{k, 2}, \widetilde{U}_{k, 3}\right\}$ of $\left(\mathcal{U} \cap Y_{k},\left.f\right|_{Y_{k}}\right)$.

Let $\widetilde{U}_{j}=\bigcup_{k=1}^{N_{1}} \widetilde{U}_{k, j}$ for each $j=1,2,3$. Then $\left\{\widetilde{U}_{1}, \widetilde{U}_{2}, \widetilde{U}_{3}\right\}$ is the desired 3 -coloring of $(X, f)$.

Lemma 3.11. Let $\left\{a_{1}, \ldots, a_{m}\right\}$ be a set of natural numbers. Then the following conditions are equivalent:

(1) There exists an $n \in \mathbb{N} \backslash\{1,3\}$ such that $a_{k}$ is a multiple of $n$ for each $k=1, \ldots, m$.

(2) $\operatorname{gcd}\left\{a_{1}, \ldots, a_{m}\right\} \neq 1,3$, where gcd is the greatest common divisor.

Proof. $(2) \Rightarrow(1)$. Put $n=\operatorname{gcd}\left\{a_{1}, \ldots, a_{m}\right\}$. Then clearly $n$ satisfies condition (1).

$(1) \Rightarrow(2)$. Let $n$ witness condition (1). Notice that $a_{k} \geq n>1$ for each $k=1, \ldots, m$, and so $\operatorname{gcd}\left\{a_{1}, \ldots, a_{m}\right\} \neq 1$. Assume to the contrary that $\operatorname{gcd}\left\{a_{1}, \ldots, a_{m}\right\}=3$. Since $n \neq 1,3$ and $n \leq 3$, it follows that $n=2$, and so 2 and 3 are common divisors of $\left\{a_{1}, \ldots, a_{m}\right\}$. Thus, $\operatorname{gcd}\left\{a_{1}, \ldots, a_{m}\right\} \geq 6$, a contradiction.

Let $\operatorname{Per}(f)=\left\{n_{x} \mid x \in \mathrm{P}(f)\right\}$. By Proposition 3.10 and Lemma 3.11, we conclude the following.

Theorem 3.12. Let $f: X \rightarrow X$ be a fixed-point free homeomorphism on a finite connected graph $X$ with $\mathrm{P}(f) \neq \emptyset$. If $\operatorname{gcd}(\operatorname{Per}(f)) \neq 1,3$, then $\operatorname{col}(X, f)=3$.

Corollary 3.13. Let $X$ be a finite connected graph and $f: X \rightarrow X$ a fixed-point free homeomorphism. If there exists an $m \in \mathbb{N} \backslash\{1,3\}$ such that $f^{p}(x) \neq x$ with $1 \leq p<m$ and $f^{m}(x)=x$ for each $x \in X$, then $\operatorname{col}(X, f)=3$. 
Corollary 3.14. Let $X$ be a finite connected graph and $f: X \rightarrow X a$ fixed-point free homeomorphism. Then $\operatorname{col}(X, f)=3$ if either of the following conditions is satisfied:

(1) $\operatorname{Per}(f)$ consists of even numbers.

(2) $\operatorname{Per}(f)$ consists of powers of some prime number $p$ with $p \neq 3$.

EXAMPLE 3.15. (1) Let $R_{n}: \mathbb{S}^{1} \rightarrow \mathbb{S}^{1}$ be the rotation through $2 \pi / n$ for each $n \in \mathbb{N}$. If $n \neq 1,3$, by Corollary 3.13 , we have $\operatorname{col}\left(\mathbb{S}^{1}, R_{n}\right)=3$. On the other hand, by Theorem 1.2 and Proposition $2.1, \operatorname{col}\left(\mathbb{S}^{1}, R_{3}\right)=4$.

(2) Let $Z_{4} * Z_{4}$ be as in Example 2.3. For any fixed-point free homeomorphism $f: Z_{4} * Z_{4} \rightarrow Z_{4} * Z_{4}$, each vertex has an even period. In fact, let $Z_{4, i}=Z_{4}$ for $i=0,1$ and $x \in Z_{4,0}$. First, assume that $f(x) \in Z_{4,0}$. If $f^{2}(x) \in Z_{4,1}$, then by finitely many iterations of $f$, we can find a simplex $x * f(x)$, which is a contradiction. This implies that $\left\{f^{n}(x) \mid n \geq 0\right\} \subset Z_{4,0}$, and thus $n_{x} \in\{2,3,4\}$. Now, if $x$ is a period 3 point, then we can take a $y \in Z_{4,0} \backslash\left\{x, f(x), f^{2}(x)\right\}$ such that $f(y) \in Z_{4,1}$. Then the following two cases can occur: (i) $f^{2}(y)=y$, (ii) $f^{2}(y) \in Z_{4,1}$. Assuming (i), we note that $f(y * f(y))=y * f(y)$, and hence $f$ has a fixed point, which is impossible. On the other hand, (ii) ensures that there exists a simplex $f(y) * f^{2}(y)$ with $\left\{f(y), f^{2}(y)\right\} \subset Z_{4,1}$, a contradiction again. Hence, $x$ has an even period.

Next, assume that $f(x) \in Z_{4,1}$. Repeating the same arguments, we can verify that $x$ has an even period $n_{x}$ with $n_{x}=4,6,8$.

Thus, every vertex of $Z_{4} * Z_{4}$ has an even period. Therefore, by Corollary 3.14 , we have $\operatorname{col}\left(Z_{4} * Z_{4}, f\right)=3$. This shows that the condition that $\operatorname{col}(X, f) \leq n+3$ for any fixed-point free homeomorphism $f: X \rightarrow X$ does not imply $\operatorname{dim} X \leq n$.

\section{References}

[1] J. M. Aarts, R. J. Fokkink and H. Vermeer, Variations on a theorem of Lusternik and Schnirelmann, Topology 35 (1996), 1051-1056.

[2] - , - - C Coloring maps of period three, Pacific J. Math. 202 (2002), 257-266.

[3] A. Błaszczyk and K. D. Yong, A topological version of a combinatorial theorem of Katětov, Comment. Math. Univ. Carolin. 29 (1988), 657-663.

[4] N. G. de Bruijn and P. Erdős, A colour problem for infinite graphs and a problem in the theory of relations, Nederl. Akad. Wetensch. Proc. Sect. A 54 (1951), 369-373.

[5] E. K. van Douwen, $\beta X$ and fixed-point free maps, Topology Appl. 51 (1993), 191-195.

[6] M. A. van Hartskamp and J. Vermeer, On colorings of maps, ibid. 73 (1996), 181-190.

[7] M. Katětov, A theorem on mappings, Comment. Math. Univ. Carolin. 8 (1967), 431-433. 
[8] J. van Mill, Easier proofs of coloring theorems, Topology Appl. 97 (1999), 155-163.

Yuji Akaike

Kure National College of Technology

2-2-11 Aga-Minami Kure-shi

Hiroshima 737-8506, Japan

E-mail: akaike@kure-nct.ac.jp

Kazuo Tomoyasu

Miyakonojo National College of Technology

473-1 Yoshio-cho Miyakonojo-shi

Miyazaki 885-8567, Japan

E-mail: tomoyasu@cc.miyakonojo-nct.ac.jp
Naotsugu Akaike Hiroshima Institute of Technology 2-1-1 Miyake, Saeki-ku Hiroshima 731-5193, Japan E-mail: naochin@cc.it-hiroshima.ac.jp

Received April 17, 2008;

received in final form February 2, 2009 